\title{
Beyond evidence: the micropolitics of improvement
}

\author{
Ann Langley, ${ }^{1}$ Jean-Louis Denis ${ }^{2}$
}

${ }^{1}$ HEC Montréal, chemin de la Côte-Ste-Catherine, Montréal, Quebec, Canada 2École Nationale d'Administration Publique, Montreal, Quebec, Canada

Correspondence to Professor Ann Langley, HEC Montréal, 3000, chemin de la Côte-Ste-Catherine, Montréal, QC, H3T 2A7, Canada; ann.langley@hec.ca

Accepted 17 October 2010

\section{ABSTRACT}

This paper aims to draw attention to the social and micropolitical dimensions of attempting to implement improvements within healthcare organisations. It is argued that quality improvement initiatives, like other forms of organisational innovation, will fail unless they are conceived and implemented in such a way as to take into account the pattern of interests, values and power relationships that surround them. Drawing on examples, it is suggested that innovators can intervene more successfully if they understand how the benefits and costs of interventions are likely to be distributed among stakeholders within their setting, how different but equally legitimate value sets may structure peoples' understanding of them and how the nature of the interventions themselves (and, in particular, the shape of their hard core and soft periphery) might provide scope for redesigning or adapting interventions in ways that are likely to make them both more effective and politically feasible.

This paper argues that however rational and reasonable they may appear on paper, quality improvement initiatives, like other forms of organisational innovation, will fail unless they are designed and implemented in such a way as to take into account the pattern of interests, values and power relationships that surround them. Would-be innovators thus need to look beyond evidence in order to make desirable initiatives more 'powerful' or influential. In other words, champions of innovation and rational process improvement need-perhaps paradoxically-to become more political.

Several authors have attempted to understand the success of quality improvement initiatives by studying organisations that have been more or less successful at implementing them. For example, Ferlie and Shortell emphasise four groups of success factors for quality improvement: leadership at all levels, a culture supportive of learning and change, teamwork and appropriate information systems. ${ }^{1}$ Other researchers have added structural, political and strategic factors. $^{2}{ }^{3}$ Similarly, Sexton, Pronovost and colleagues point to the importance of clinical culture in successfully implementing patient safety initiatives. ${ }^{45}$ These ideas all make sense, and organisations clearly need to invest in these areas to improve their performance.

However, knowledge of these general factors unfortunately does not tell us much about how and why any specific evidencebased innovation or improvement initiative does or does not receive support in a specific context. This is an important practical question. As we know from extensive research, formal evidence concerning the superiority of any practice is insufficient in itself to ensure its implementation. ${ }^{67}$ In his treatise on how to "make social science matter,' Bent Flyvbjerg notes that knowledge of power relations in any given context is necessary to understand what is taken to be rational and right, and moreover that rationality (eg, research 'evidence') can prevail only if it takes power into account. ${ }^{8} 9$ In this paper, we elaborate on this idea and consider its practical implications for those who wish to move quality improvement initiatives into practice.

Specifically, we introduce and illustrate here three key observations based on our previous research on the diffusion and adoption processes of complex healthcare innovations that involve both clinical and management stakeholders. ${ }^{10}$ Many quality improvement initiatives are of this type. We also relate these observations to research by other scholars who have looked at change in healthcare practice from a managerial perspective. $^{2}{ }^{3} 7$ For each observation, we first present the central point, then provide an example from our research and finally, explore its practical implications.
This paper is freely available Journals unlocked scheme, see http://qualitysafety.bmj. com/site/about/unlocked. xhtml 


\section{OBSERVATION 1: THE DISTRIBUTED NATURE OF COSTS AND BENEFITS}

The first observation is that complex interventions aimed at implementing new healthcare technologies or at improving quality tend to have distributed costs and benefits. In other words, while an innovation might theoretically have potential benefits for patients, the consequences may not be uniformly positive for all the other people involved in its implementation.

One of the cases we looked at in our research involved the use of low-molecular-weight heparin (LMWH) to treat deep vein thrombosis. ${ }^{10}$ At the time of the study, there was plenty of scientific evidence that the procedure was safe and valuable for patients. ${ }^{11}$ However, this innovation had different implications for different stakeholders. For hospital administrators, it was a way to save beds because the treatment allowed early discharge and follow-up in the community. For some doctors, it was seen as 'best practice' and a way to increase throughput (and possibly income), while for others, it raised concern that patients might not be adequately followed up (as well as leading to reduced income). For nurses, it meant more time spent on teaching to enable patients to inject themselves. For community clinics, it involved increased responsibilities that were not necessarily covered by increased budgets. For patients, it could be a way to avoid hospitalisation, or perhaps an increased cost and inconvenience. Negotiating across interprofessional and interorganisational lines further made convergence complicated, although the diverse benefits for a variety of actors provided multiple entry points in the push to build a coalition in favour of adoption.

The point is that the distributed nature of the benefits and costs of any practice needs to be understood if one wants to implement change. Although some quality improvement proponents might feel uncomfortable stepping outside the role of rational expert, knowing 'what's in it for whom' is helpful in seeing how and why some new practices might meet with resistance, while others are adopted very easily. (Sometimes, innovations may even be adopted too easily. A corollary of the argument above is that when a new practice immediately seems to be in everyone's interest, there can be a rush to adopt it, sometimes before clear evidence is available, as may have happened, for example, during the early years of laparoscopic surgery. ${ }^{10}$ ) A useful tool for assessing the weight of support and likely lines of resistance for a given intervention project is 'stakeholder mapping. ${ }^{12}$ This involves placing key stakeholders on a two-by-two grid showing (a) their power to influence adoption and (b) their interest in doing so. For example, elements that might be used to position stakeholders according to their relative power might include assessments of formal authority, control of resources (expertise, budgets, prestige) and connections to powerful others (eg, senior management, media, etc). To assess interests, one can examine the likely costs and benefits of the focal project for that stakeholder. Such an analysis can be helpful in understanding whether, when and why the project is likely to receive support, and in thinking about strategies for coalition-building, a crucial step in achieving organisational change. ${ }^{13} 14$

\section{OBSERVATION 2: THE VARIETY OF VALUE SYSTEMS UNDERLYING INTERVENTIONS}

Our second observation is that the people involved in implementing quality improvements hold different values about what is appropriate that may underpin particular interests. While interests revolve around personal benefits (what is in this for me personally?), values concern what is right and good. People naturally tend to justify their positions in terms of such legitimate concerns. Indeed, different professional groups and providers tend to see the world in different ways in correspondence with their training and experience. ${ }^{7}$ For example, while evidence represented by randomised control trials may be the gold standard in determining appropriate care for an academic health centre physician, issues such as equity, cost and patient choice may quite legitimately underlie the positions of other stakeholders such as administrators and community groups. Sometimes these different value sets appear incommensurable, and explain why agreement is so difficult despite apparently incontrovertible evidence.

For example, one of the innovations examined in our study was 'assertive community treatment' (ACT) for psychiatric patients with severe psychotic disorders. ACT is a highly normalised intervention package involving multidisciplinary teams who take an active, even aggressive, part in supporting patients in the community. It has been extensively studied in randomised control trials and found to be effective on a long list of criteria such as quality of life, medication compliance, patient satisfaction and cost. ${ }^{15}$ However, we found strong ideologically based differences of opinion about it between those who favoured 'medical' or 'social' approaches to mental health, placing in stark contrast the 'scientific' model of traditional medicine and the 'social' model of community activists where patient freedom and the avoidance of control were preferred. ${ }^{16}$ This issue raised more passions on both sides than any other innovation we studied. ${ }^{10}$ Clearly, there was much more to this than a question of scientific evidence. And clearly, scientific evidence is not value-free.

To different degrees, value differences may underlie many potential improvement interventions. For example, 
Ferlie and colleagues described how differences in how nurses and doctors viewed evidence intervened in determining the spread of innovations. ${ }^{7}$ The very notion of 'quality' can raise fierce ideological debates between those who see it purely in terms of clinical excellence and those who see it more broadly in terms of providerpatient relationships. For example, Barker urges a return to the values of individualised caring associated traditionally with the nursing profession that he sees as being undermined by the emphasis on impersonal standardised protocols associated with evidence-based practice. ${ }^{17}$

The important thing to understand here is that when profound value differences underlie conflict about initiatives, calling on 'evidence' in a dogmatic way may simply talk past the key ethical dilemmas involved rather than addressing or confronting them. As media richness theory suggests, dealing with disagreements about goals and values requires richer information exchange involving face-to-face discussions to find room for mutual understanding. ${ }^{18} 19$

\section{OBSERVATION 3: THE HARD CORE AND THE SOFT PERIPHERY}

The third observation is that innovations or quality improvement initiatives are not as simple, clear and bounded as some of the evidence used to support them might imply. Rather, innovations tend to have a hard core and a soft periphery. ${ }^{10}$ The hard core is the element that is irreducible and that carries the key potential benefit (eg, the use of a drug). However, this is surrounded by a gamut of complementary arrangements involved in delivering the benefit that may take a variety of different forms. If the soft part of the intervention is poorly organised, it may destroy the benefit of the hard core. On the other hand, the soft periphery also offers considerable scope for organising the delivery of an innovation in a way that will ensure support from a range of stakeholders by potentially improving the cost-benefit equation for them.

For example, in the LMWH case described above, it is clearly the delivery of the drug that provides the benefit (the hard core). However, in order for this to occur, a variety of arrangements for injection and follow-up had to be designed (the soft periphery). There were multiple ways of doing this that made little difference to the clinical outcomes, but that could make a considerable difference to the distribution of benefits and costs to other stakeholders. Another of the practices we looked at involved the adoption of reusable haemodialysis filters (to be reused on the same patient). This is a practice that did not necessarily have strong clinical benefits, but it had been proven to be safe and offered financial benefits. We found in our study that the organisations that succeeded in implementing this practice were those that agreed to share the overall financial benefits with the nephrologists-for example, by enabling them to increase the number of dialysis machines in operation. ${ }^{10}$ The 'soft periphery' in this case basically concerned how the economic benefits were distributed.

The lesson here is that in attempting to implement innovations, it is important to distinguish between what is important and what is not. The soft periphery can be an ally for those who wish to intervene to improve care by providing scope for negotiation. Nevertheless, it is also true that for some innovations, distinguishing between the hard core and the soft periphery can be difficult. For example, the ACT intervention mentioned above involves a complex package of measures. Yet, the role of each component of the package in producing the proven RCT outcomes is not entirely clear. While some people argued that the only way to ensure reliable effects is to implement the entire package, others selected those elements that appeared most critical and feasible. ${ }^{10}$ The limits of 'evidence,' even RCT evidence, are evident here! Without a degree of common sense, and yes, political savwy, about what might realistically make things better, it may be that nothing will be achieved at all.

\section{DISCUSSION AND IMPLICATIONS}

This paper has attempted to reach beyond the discussion of generalised success factors to think both more theoretically and more concretely about what is going on when innovation or improvement initiatives are being considered. We argued that would-be innovators would benefit from understanding how the benefits and costs of interventions are likely to be distributed among stakeholders with different degrees of influence, how different but equally legitimate value sets may structure people's understandings of them, and how the nature of the interventions themselves (the shape of their hard core and soft periphery) might provide scope for redesigning interventions in ways that are likely to make them both effective and feasible.

Underlying these arguments is an understanding of organisations as political systems. Power in healthcare organisations is known to be diffuse as compared with most businesses because of the importance of professional expertise. Elsewhere, we have referred to these organisations as 'pluralistic settings. ${ }^{20}$ Making quality interventions work and stick in such settings requires network building and persistence. Scientific evidence can certainly contribute-many managers and professionals value it. But for all the reasons indicated above, it is insufficient on its own. A conception of healthcare organisations as political systems suggests that those who wish to improve them need, as Flyvbjerg indicates, ${ }^{9}$ to 
learn to understand how they work - not 'in general' but specifically, to think carefully about the nature of the interventions they are planning to implement and find pathways towards improvement that take the micropolitics surrounding them into account. However, before everyone rushes off to read Machiavelli, such a perspective also brings us to two complementary observations that warrant comment and reflection.

The first is a warning. Unfortunately, there is some truth to the idea that healthcare organisations sometimes seem to diffuse power among almost everyone, except the people for whom they exist-the patients needing care, especially the most vulnerable and least protected by advocacy from family and friends. There is thus a danger in an overly 'pragmatic' political perspective if it means that their voices are not heard. For this reason, bringing patients into the process needs to be encouraged. Concepts such as "patient-centred care' or 'patient empowerment' are intended to achieve this. Madison provides an insightful review of the ways in which patients may come to have influence within healthcare systems as well as the advantages, risks and political implications of such participation. ${ }^{21}$

Our final point brings us back to the observation that organisations that have been successful in consistently implementing improvement initiatives have certain characteristics: for example, they exhibit leadership at all levels, and have cultures supportive of learning and innovation, teamwork and strong information systems. ${ }^{1}$ In fact, these are all features of organisational contexts that structure political dynamics in ways that make quality issues more salient. For example, information systems that create accountabilities change the distribution of costs and benefits of innovation for individual stakeholders, supportive cultures change the values that are seen as legitimate, while teamwork ensures greater socialisation to these common values. Leadership implies the existence of people who are capable of navigating the system to get things done. The 'evidence' for factors such as these is overwhelming. ${ }^{23}$

And yet, evidence is not everything, as we have been saying all along. Those who wish to intervene to create the overall conditions described as generative of continuous quality improvement need to understand that intervening to create those conditions is also a political process. It too (and every laborious step on its way) is affected by the distribution of costs and benefits of any changes among stakeholders, by the mixture of value systems in play and by the interpretation of what is central and peripheral in the intervention itself, all this in contexts where power is diffuse, and the capacity to unilaterally impose direction is limited. Creating the conditions for continuous quality improvement is a micropolitical process all the way down.
All this suggests that knowledge about the processes of intervention and of organisational change is as important or more important to the improvement of practices and of organisations as knowledge about which interventions and organisations are most effective. Practitioners need to be sensitive to accumulating process-based knowledge, and scholars need to invest further in developing it. ${ }^{7} 10202223$

Competing interests None.

Provenance and peer review Not commissioned; externally peer reviewed.

\section{REFERENCES}

1. Ferlie EB, Shortell SM. Improving the quality of healthcare in the United Kingdom and the United States: A framework for change. Milbank Q 2001;79:281-315.

2. Baker GR, Maclntosh-Murray A, Porcellato C, et al. High Performing Healthcare Systems: Delivering Quality by Design. Toronto: Longwoods Publishing Corporation, 2008:11-26.

3. Bate P, Mendel P, Robert G. Organizing for Quality. Abingdon, UK: Radcliffe Publishing, 2008:187-201.

4. Pronovost P, Berenholtz SM, Goeschel CA, et al. Creating high reliability in health care organizations. Health Serv Res 2006;41(4 Part II):1599.

5. Sexton J, Thomas E, Pronovost P. The context of care and the patient care team: the safety attitudes questionnaire. In: Reid PP, Compton WD, Grossman JH, et al, eds. Building a Better Delivery System. A New Engineering Health Care Partnership. Washington, DC: National Academies Press, 2005:119-23.

6. Grol R, Grimshaw J. From best evidence to best practice: Effective implementation of change in patients' care. Lancet2003;362:1225-30.

7. Ferlie EB, Fitzgerald L, Wood M, et al. The non spread of innovations: The mediating role of professionals. Acad Manage $J$ 2005;48:117-34.

8. Flyvbjerg B. Rationality and Power: Democracy in Practice. Chicago: University of Chicago Press, 1998.

9. Flyvbjerg B. Making Social Science Matter. Cambridge: Cambridge University Press, 2001.

10. Denis JL, Hébert $Y$, Langley A, et al. Explaining diffusion patterns for complex health care innovations. Health Care Manage Rev 2002;27:60-73.

11. Levine M, Gent M, Hirsch J, et al. A comparaison of low-molecularweight heparin administered at home with unfractionated heparin administered in the hospital for proximal deep-vein thrombosis. N Engl J Med 1996;334:677-81.

12. Scholes K. Stakeholder mapping: A practical tool for public sector managers. In: Johnson G, Scholes K, eds. Exploring Public Sector Strategy. London: Prentice-Hall, 2001:165-84.

13. Kotter JP. Leading Change. Cambridge, MA: Harvard Business School Press.

14. Bate $P$, Robert $G$, Bevan $H$. The next phase of healthcare improvement: what can we learn from social movements. Qual Saf Health Care 2004;13:62-6.

15. Burns BJ, Santos AB. Assertive community treatment: an update of randomized trials. Psychiatr Serv 1995;46:669-75.

16. Gomory T. The origins of coercion in assertive community treatment A review of early publications from the special treatment unit of Mendota State Hospital. Ethical Hum Sci Serv 2002;4:3-16.

17. Barker P. Reflections on caring as a virtue ethic in an evidence-based culture. Int J Nurs Stud 2000;37:329-36.

18. Daft $\mathrm{RL}$, Lengel $\mathrm{RH}$. Organizational information requirements, media richness and structural design. Manage Sci 1986;32:554-71.

19. Thomas JB, Trevino LK. Information-processing in strategic alliance building - a multiple-case approach. J Manage Stud 1993;30:779-814.

20. Denis JL, Lamothe L, Langley A. The dynamics of collective leadership and strategic change in pluralistic organizations. Acad Manage J 2001;44:809-37.

21. Madison K. Patients as 'regulators'? Patients' evolving influence over health care delivery. J Leg Med 2010;31:9-34.

22. Greenhalgh T, Stones R. Theorising big IT programs in healthcare: Strong structuration theory meets actor-network theory. Soc Sci Med 2010;70:1285-94.

23. Buchanan DA, Fitzgerald L, Ketley D. The Sustainability and Spread of Organizational Changes: Modernizing Healthcare. Abingdon, UK: Routledge, 2007. 